\title{
Speeding up constrained path solvers with a reachability propagator
}

\author{
Luis Quesada, Peter Van Roy, and Yves Deville \\ Université catholique de Louvain \\ Place Sainte Barbe, 2, B-1348 Louvain-la-Neuve, Belgium \\ \{luque, pvr, yde\}@info.ucl.ac.be
}

\begin{abstract}
Constrained path problems have to do with finding paths in graphs subject to constraints. One way of constraining the graph is by enforcing reachability on nodes. For instance, it may be required that a node reaches a particular set of nodes by respecting some restrictions like visiting a particular set of nodes or edges and using less than a certain amount of resources. The reachability constraints of this paper were suggested by a practical problem regarding mission planning in the context of an industrial project.

We deal with this problem by using concurrent constraint programming where the problem is solved by interleaving Propagation and Labeling. In this paper, we define a propagator which we call Reachability that implements a generalized reachability constraint on a directed graph $g$. Given a source node source in $g$, we can identify three parts in the Reachability constraint: (1) the relation between each node of $g$ and the set of nodes that it reaches, (2) the association of each pair of nodes $\langle$ source, $i\rangle$ with its set of cut nodes, and (3) the association of each pair of nodes $\langle$ source, $i\rangle$ with its set of bridges.

We show the effectiveness of our Reachability propagator by applying it to the Simple Path problem with mandatory nodes. We do an experimental evaluation of Reachability that shows that it provides strong pruning, obtaining solutions with very little search. Furthermore, we show that Reachability is also useful for defining a good labeling strategy and dealing with ordering constraints among mandatory nodes. These experimental results give evidence that Reachability is a useful primitive for solving constrained path problems over graphs.
\end{abstract}

\section{Introduction}

Constrained path problems have to do with fi nding paths in graphs subject to constraints. One way of constraining the graph is by enforcing reachability on nodes. For instance, it may be required that a node reaches a particular set of nodes by respecting some restrictions like visiting a particular set of nodes or edges and using less than a certain amount of resources. We have instances of this problem in Vehicle routing [14, 4, 9] and Bioinformatics[7].

An approach to solve this problem is by using Concurrent Constraint Programming (CCP) [18, 13]. In $\mathrm{CCP}$, we solve the problem by interleaving two processes: propagation and labeling. In Propagation, we are interested in fi ltering the domains of a set of fi nite domain variables according to the semantics of the constraints that have to be respected. In Labeling, we are interested in specifying which alternative should be selected when searching for the solution.

Our goal is to implement so-called Constrained Path Propagators (CPPs) for achieving global consistency [6]. In this paper, we defi ne a propagator which we call Reachability that implements a generalized reachability constraint on a directed graph $g$. Given a source node source in $g$, we can identify three parts in the Reachability constraint: (1) the relation between each node of $g$ and the set of nodes that it reaches, (2) the association of each pair of nodes $\langle$ source, $i\rangle$ with its set of cut nodes, and (3) the association of each pair of nodes $\langle$ source, $i\rangle$ with its set of bridges. 


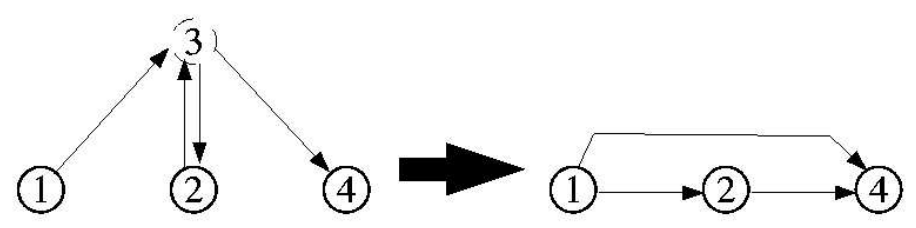

Figure 1: Relaxing Simple Path with mandatory nodes by eliminating the optional nodes

Our contribution is a propagator that is suitable for solving the Simple Path problem with mandatory nodes $[19,3]$. This problem consists in fi nding a simple path in a directed graph containing a set of mandatory nodes. A simple path is a path where each node is visited once. Certainly, this problem can be trivially solved if the graph has no cycles since in that case there is only one order in which we can visit the mandatory nodes [19]. However, if the graph has cycles the problem is NP complete since we can easily reduce the Hamiltonian Path problem [10,5] to this problem.

Notice however that we can not trivially reduce Simple Path with mandatory nodes to Hamiltonian path. One could think that optional nodes (i.e. nodes that are not mandatory) can be eliminated in favor of new edges as a preprocessing step, which fi nds a path between each pair of mandatory nodes. However, the problem is that the paths that are precomputed may share nodes. This may lead to violations of the requirement that a node should be visited only once.

In fi gure 1, we illustrate this situation. Mandatory nodes are in solid lines. In the second graph we have eliminated the optional nodes by connecting each pair of mandatory nodes depending on whether there is a path between them. However, we observe that the second graph has a simple path going from node 1 to node 4 (visiting all the mandatory nodes) while the fi rst one does not. Indeed, the simple path in the second graph is not a valid solution to the original problem since it implies that node 3 is visited twice.

The other reason that makes the elimination of optional nodes diffi cult is that fi nding $k$ pairwise disjoint paths between $k$ pairs of nodes $\left\langle s_{1}, d_{1}\right\rangle,\left\langle s_{2}, d_{2}\right\rangle, \ldots,\left\langle s_{k}, d_{k}\right\rangle$ is NP complete [20].

In general, we can say that the set of optional nodes that can be used when going from a mandatory node $a$ to a mandatory node $b$ depends on the path that has been traversed before reaching $a$. This is because the optional nodes used in the path going from the source to $a$ can not be used in the path going from $a$ to $b$.

From our experimental measurements in Section 4, we observe that the suitability of Reachability for dealing with Simple Path with mandatory nodes is based on the following aspects:

- The strong pruning that Reachability performs. Due to the computation of cut nodes and bridges (i.e., nodes and edges that are present in all the paths going from a given node to another), Reachability is able to discover non-viable successors early on.

- The information that Reachability provides for implementing smart labeling strategies. By labeling strategy we mean the way the search tree is created, i.e., which constraint is used for branching. Reachability associates each node with the set of nodes that it reaches. This information can be used to guide the search in a smart way. For instance, one of our observations is that, when choosing first the node $i$ that reaches the most nodes and selecting as a successor of $i$ fi rst a node that $i$ reaches, we obtain paths that minimize the use of optional nodes.

An additional feature of Reachability is its suitability for imposing ordering constraints among mandatory nodes (which is a common issue in routing problems). In fact, it might be the case that we have to visit the nodes of the graph in a particular (partial) order. Taking into account that a node $a$ reaches a node $b$ if 
there is a path going from node $a$ to node $b$, we force a node $i$ to be visited before a node $j$ by imposing that $i$ reaches $j$ and $j$ does not reach $i$. We have performed experiments that show that Reachability takes the most advantage of this information to avoid branches in the search tree with no solution.

The structure of the paper is as follows: fi rst, we introduce Reachability by presenting its semantics and deriving pruning rules in a systematic way. Then, we show how we can model Simple Path with mandatory nodes in terms of Reachability. Finally, we show examples that demonstrate the performance of Reachability for this type of problem, and elaborate on some works that are related to our approach.

\section{The reachability propagator}

\subsection{Reachability constraint}

The Reachability Constraint is defi ned as follows:

$$
\begin{aligned}
& r n(i)=\operatorname{Reach}(g, i) \wedge \\
& \text { Reachability }(g, \text { source, } r n, c n, b e) \equiv \forall_{i \in N} \cdot \operatorname{cn}(i)=C u t N o d e s(g, \text { source }, i) \wedge \\
& \text { be }(i)=\operatorname{Bridges}(g, \text { source, } i)
\end{aligned}
$$

Where:

- $g$ is a graph whose set of nodes is a subset of $N$.

- source is a node of $g$.

- $r n(i)$ is the set of nodes that $i$ reaches.

- $c n(i)$ is the set of nodes appearing in all paths going from source to $i$.

- be $(i)$ is the set of edges appearing in all paths going from source to $i$.

- Reach, Paths, CutNodes and Bridges are functions that can be formally defi ned as follows:

$$
\begin{gathered}
j \in \operatorname{Reach}(g, i) \leftrightarrow \exists_{p} \cdot p \in \operatorname{Paths}(g, i, j) \\
p \in \operatorname{Paths}(g, i, j) \leftrightarrow \begin{array}{l}
p=\left\langle k_{1}, \ldots, k_{h}\right\rangle \in \operatorname{nodes}(g)^{h} \wedge k_{1}=i \wedge k_{h}=j \wedge \\
\forall_{1 \leq f<h .} .\left\langle k_{f}, k_{f+1}\right\rangle \in \operatorname{edges}(g)
\end{array} \\
k \in \text { CutNodes }(g, i, j) \leftrightarrow \forall_{p \in \operatorname{Paths}(g, i, j)} \cdot k \in \operatorname{nodes}(p) \\
e \in \operatorname{Bridges}(g, i, j) \leftrightarrow \forall_{p \in \operatorname{Paths}(g, i, j)} \cdot \operatorname{e} \in \operatorname{edges}(p)
\end{gathered}
$$

The above defi nition of Reachability implies the following properties which are crucial for the pruning that Reachability performs. These properties defi ne relations between the functions $r n, c n$, be, nodes and edges. These relations can then be used for pruning, as we show in section 2.2.

1. If $\langle i, j\rangle$ is an edge of $g$, then $i$ reaches $j$.

$$
\forall\langle i, j\rangle \in \operatorname{edges}(g) \cdot j \in r n(i)
$$


2. If $i$ reaches $j$, then $i$ reaches all the nodes that $j$ reaches.

$$
\forall_{i, j, k \in N} \cdot j \in r n(i) \wedge k \in r n(j) \rightarrow k \in r n(i)
$$

3. If source reaches $i$ and $j$ is a cut node between source and $i$ in $g$, then $j$ is reached from source and $j$ reaches $i$ :

$$
\forall_{i, j \in N} . i \in \operatorname{rn}(\text { source }) \wedge j \in \operatorname{cn}(i) \rightarrow j \in \operatorname{rn}(\text { source }) \wedge i \in \operatorname{rn}(j)
$$

4. Reached nodes, cut nodes and bridges are nodes and edges of $\mathrm{g}$ :

$$
\forall_{i \in N} \cdot r n(i) \subseteq \operatorname{nodes}(g) \quad(9) \quad \forall_{i \in N} . c n(i) \subseteq \operatorname{nodes}(g) \quad(10) \quad \forall_{i \in N} . b e(i) \subseteq \operatorname{edges}(g)
$$

\subsection{Pruning rules}

We implement the constraint in Equation 1 with the propagator

$$
\text { Reachability }(G, \text { Source, } R N, C N, B E)
$$

In this propagator we have that:

- $G$ is a graph variable (i.e., a variable whose domain is a set of graphs [8]). The upper bound of $G$ $(\max (G))$ is the greatest graph to which $G$ can be instantiated, and its lower bound $(\min (G))$ is the smallest graph to which $G$ can be instantiated. So, $i \in \operatorname{nodes}(G)$ means $i \in \operatorname{nodes}(\min (G))$ and $i \notin \operatorname{nodes}(G)$ means $i \notin \operatorname{nodes}(\max (G))$ (the same applies for edges). In what follows, $\left\{\left\langle N_{1}, E_{1}\right\rangle \#\left\langle N_{2}, E_{2}\right\rangle\right\}$ will denote a graph variable whose lower bound is $\left\langle N_{1}, E_{1}\right\rangle$ and upper bound is $\left\langle N_{2}, E_{2}\right\rangle$. I.e., if $g=\langle n, e\rangle$ is the graph that $G$ approximates, then $N_{1} \subseteq n \subseteq N_{2}$ and $E_{1} \subseteq e \subseteq E_{2}$.

- Source is an integer representing the source in the graph.

- $R N(i)$ is a Finite Integer Set (FS) [11] variable associated with the set of nodes that can be reached from node $i$. The upper bound of this variable $(\max (R N(i)))$ is the set of nodes that could be reached from node $i$ (i.e., nodes that are not in the upper bound are nodes that are known to be unreachable from $i$ ). The lower bound $(\min (R N(i)))$ is the set of nodes that are known to be reachable from node $i$. In what follows $\left\{S_{1} \# S_{2}\right\}$ will denote a FS variable whose lower bound is the set $S_{1}$ and upper bound is the set $S_{2}$.

- $C N(i)$ is a FS variable associated with the set of nodes that are included in every path going from Source to $i$.

- $B E(i)$ is a FS variable associated with the set of edges that are included in every path going from Source to $i$.

The defi nition of Reachability and its derived properties give place to a set of propagation rules. We show here the most representative ones. The others are given in [16]. A propagation rule is defi ned as $\frac{C}{A}$ where $C$ is a condition and $A$ is an action. If $C$ is true, the pruning defi ned by $A$ can be performed.

- From (6) $\forall_{\langle i, j\rangle \in \operatorname{edges}(g)} \cdot j \in r n(i)$ we obtain:

$$
\frac{\langle i, j\rangle \in \operatorname{edges}(\min (G))}{j \in \min (R N(i))}
$$


- From (7) $\forall_{i, j, k \in N} \cdot j \in r n(i) \wedge k \in r n(j) \rightarrow k \in r n(i)$ we obtain:

$$
\frac{j \in \min (R N(i)) \wedge k \in \min (R N(j))}{k \in \min (R N(i))}
$$

- From (8) $\forall_{i, j \in N} . i \in r n($ source $) \wedge j \in c n(i) \rightarrow j \in r n($ source $) \wedge i \in r n(j)$ we obtain:

$$
\begin{aligned}
& \frac{i \in \min (R N(\text { Source })) \wedge j \in \min (C N(i))}{j \in \min (R N(\text { Source }))} \\
& \frac{i \in \min (R N(\text { Source })) \wedge j \in \min (C N(i))}{i \in \min (R N(j))}
\end{aligned}
$$

- From $(1) \forall_{i \in N} \cdot r n(i)=\operatorname{Reach}(g, i)$ we obtain:

$$
\frac{j \notin \operatorname{Reach}(\max (G), i)}{j \notin \max (R N(i))}
$$

- From (1) $\forall_{i \in r n(\text { source }) . c n}(i)=C u t N o d e s(g$, source, $i)$ we obtain:

$$
\frac{j \in C u t N o d e s(\max (G), \text { Source }, i)}{j \in \min (C N(i))}
$$

- From (1) $\forall_{i \in \text { rn (source) }} . b e(i)=\operatorname{Bridges}(g$, source, $i)$ we obtain:

$$
\frac{e \in \operatorname{Bridges}(\max (G), \text { Source }, i)}{e \in \min (B E(i))}
$$

- From (9) $\forall_{i \in N} \cdot r n(i) \subseteq \operatorname{nodes}(g),(10) \forall_{i \in N} \cdot c n(i) \subseteq \operatorname{nodes}(g)$ and (11) $\forall_{i \in N} \cdot b e(i) \subseteq$ edges $(g)$ we obtain:

$$
\frac{k \in \min (R N(i))}{k \in \operatorname{nodes}(\min (G))} \quad \text { (20) } \quad \frac{k \in \min (C N(i))}{k \in \operatorname{nodes}(\min (G))} \quad \text { (21) } \quad \frac{e \in \min (B E(i))}{e \in \operatorname{edges}(\min (G))}
$$

\subsection{Implementation of Reachability}

Reachability has been implemented using a message passing approach [21] on top of the multi-paradigm programming language $\mathrm{Oz}$ [12]. In [15], we discuss the implementation of Reachability in detail. In this section we will simply refer to some of the functions that are used in the implementatin of Reachability:

In our pruning rules we have three functions:

- Reach that is $O(N+E)$ since it is basically a call to DFS [5].

- CutNodes whose algorithm is based on the following defi nition:

$$
k \in C u t N o d e s(g, i, j) \leftrightarrow j \notin \operatorname{Reach}(\operatorname{RemoveNode}(g, k), i)
$$

So, checking whether a node is a cut node is $O(N+E)$. Notice that we assume that RemoveNode returns the same graph when $k \notin \operatorname{nodes}(g)$. 
- Bridges whose algorithm is based on the following defi nition:

$$
e \in \operatorname{Bridges}(g, i, j) \leftrightarrow j \notin \operatorname{Reach}(\text { RemoveEdge }(g, e), i)
$$

So, checking whether an edge is a bridge is $O(N+E)$. Notice that we assume that RemoveEdge returns the same graph when $e \notin e$ edges $(g)$.

The computation of cut nodes and bridges in each propagation step implies running DFS per each potential cut node and per each potential bridge. However, we take advantage of the fact that the cut nodes and the bridges are part of the tree returned by DFS (assuming that the destination node is reached from the source). In fact, this means that both the computation of cut nodes and the computation of bridges have the same complexity since the number of edges in the DFS tree is proportional to the number of nodes. I.e., updating $C N / B E$ after removing a set of edges from the upper bound of $G$ is $O(N *(N+E))^{1}$.

As explained in [15], we do not compute cut nodes and bridges each time an edge is removed since this certainly leads to a considerably amount of unnecessary computation. This is due to the fact that the set of cut nodes/bridges evolves monotonically. What we actually do is to consider all the removals at once and make one computation of cut nodes and bridges per set of edges removed.

\section{Solving Simple Path with mandatory nodes with Reachability}

In this section we will elaborate on the important role that Reachability can play in solving Simple Path with mandatory nodes. This problem consists in fi nding a simple path in a directed graph containing a set of mandatory nodes. A simple path is a path where each node is visited once. I.e., given a directed graph $g$, a source node source, a destination node dest, and a set of mandatory nodes mandnodes, we want to fi nd a path in $g$ that goes from source to dest, going through mandnodes and visiting each node only once.

The contribution of Reachability consists in discovering nodes/edges that are part of the path early on. This information is obtained by computing the cut nodes and bridges in each labeling step. Let us consider the following two cases ${ }^{2}$ :

- Consider the graph variable on the left of Figure 2. Assume that node 1 reaches node 9. This information is enough to infer that node 5 belongs to the graph, node 1 reaches node 5 , and node 5 reaches node 9.

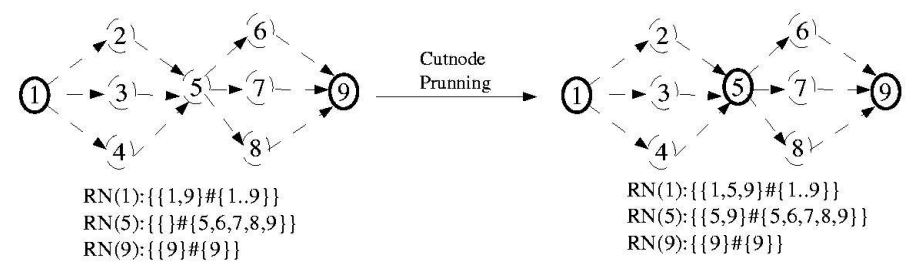

Figure 2: Discovering cut nodes

\footnotetext{
${ }^{1}$ Notice that the notion of cut node that we have presented does not correspond to the notion of articulation point. An articulation point is a node whose removal increases the number of strongly connected components. A cut node, in our defi nition, does not necessarily have this property.

${ }^{2}$ In Figures 2 and 3, nodes and edges that belong to the lower bound of the graph variable are in solid line. For instance, the graph variable on the left side of Figure 2 is a graph variable whose lower bound is the graph $\langle\{1,5\}, \emptyset\rangle$, and whose upper bound is the graph $\langle\{1,2,3,4,5,6,7,8,9\},\{\langle 1,2\rangle,\langle 1,3\rangle,\langle 1,4\rangle,\langle 2,5\rangle,\langle 3,5\rangle,\langle 4,5\rangle,\langle 5,6\rangle,\langle 5,7\rangle,\langle 5,8\rangle,\langle 6,9\rangle,\langle 7,9\rangle,\langle 8,9\rangle\}\rangle$.
} 
- Consider the graph variable on the left of Figure 3. Assume that node 1 reaches node 5. This information is enough to infer that edges $\langle 1,2\rangle,\langle 2,3\rangle,\langle 3,4\rangle$ and $\langle 4,5\rangle$ are in the graph, which implies that node 1 reaches nodes $1,2,3,4,5$, node 2 at least reaches nodes $2,3,4,5$, node 3 at least reaches nodes $3,4,5$ and node 4 at least reaches nodes 4,5 .

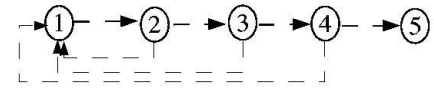

$\mathrm{RN}(1):\{\{1,5\} \#\{1.5\}\}$ $\mathrm{RN}(2):\{\{\} \#\{1 . .5\}\}$ $\operatorname{RN}(3):\{\{\} \#\{1 . .5\}$ $\operatorname{RN}(4):\{\{\} \#\{1 . .5\}\}$ $\mathrm{RN}(5):\{\{5\} \#\{5\}\}$

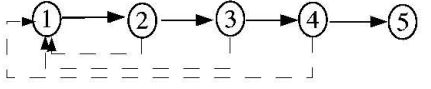

$\operatorname{RN}(1):\{\{1.5\} \#\{1.5\}\}$ $R N(2):\{\{2 . .5\} \#\{1.5\}\}$ $\operatorname{RN}(3):\{\{3 . .5\} \#\{1.5\}\}$ $\operatorname{RN}(4):\{\{4,5\} \#\{1 . .5\}\}$ $\operatorname{RN}(5):\{\{5\} \#\{5\}\}$

Figure 3: Discovering bridges

Notice that Hamiltonian Path (i.e., the problem where we have to fi nd a simple path between two nodes containing all the nodes of the graph $[10,5]$ ) can be reduced to Simple Path with mandatory nodes by defi ning the set of mandatory nodes as nodes $(g)-\{$ source, dest $\}$.

The above defi nition of Simple Path with mandatory nodes can be formally defi ned as follows:

$$
\begin{aligned}
& p \in \operatorname{Paths}(g, \text { source }, \text { dest }) \wedge \\
& \operatorname{SPMN}(g, \text { source }, \text { dest }, \text { mandnodes }, p) \leftrightarrow \quad \operatorname{Nocle}(p) \wedge \\
& \text { mandnodes } \subset \operatorname{nodes}(p)
\end{aligned}
$$

$S P M N$ stands for "Simple Path with mandatory nodes". NoCycle $(p)$ states that $p$ is a simple path (i.e., a path where no node is visited twice). This defi nition of Simple Path with mandatory nodes implies the following property:

$$
\text { Reachability }(p, \text { source, } r n, c n, \text { be }) \wedge \text { dest } \in \operatorname{rn}(\text { source }) \wedge \text { cn }(\text { dest }) \supseteq \text { mandnodes }
$$

This is because the destination is reached by the source and the path contains the mandatory nodes. This derived property and the fact that we can implement $S P M N$ in terms of the AllDiff constraint [17] and the NoCycle constraint [4] suggest the two approaches for Simple Path with mandatory nodes summarized in Table 1 (which are compared in the next section). In the fi rst approach, we basically consider AllDiff and NoCycle. In the second approach we additionally consider Reachability.

Notice that, even though the computation of bridges plays a crucial role in the pruning that Reachability performs, we do not use the be argument in the second approach. In fact be can play an important role in solving Constrained Euler Path problems (i.e., problems where the objective is to fi nd a path visiting a set of edges by respecting some additional constraints).

\begin{tabular}{|l|l|}
\hline Approach 1 & Approach 2 \\
\hline$S P M N(g$, source, dest, mandnodes,$p)$ & $S P M N(g$, source, dest, mandnodes,$p)$ \\
& Reachability $(p$, source,$r n$, cn, be $)$ \\
& dest $\in$ rn $($ source $)$ \\
& cn $($ dest $) \supseteq$ mandnodes \\
\hline
\end{tabular}

Table 1: Two approaches for solving Simple Path with mandatory nodes 


\section{Experimental results}

In this section we present a set of experiments that show that Reachability is suitable for Simple Path with mandatory nodes. I.e., in our experiments Approach 2 (in Table 1) outperforms Approach 1. These experiments also show that Simple Path with mandatory nodes tends to be harder when the number of optional nodes increases if they are uniformly distributed in the graph. We have also observed that the labeling strategy that we implement with Reachability tends to minimize the use of optional nodes (which is a common need when the resources are limited).

In Table 2, we defi ne the instances on which we made the tests of Table 4. The id of the destination is also the size of the graph. The column Order is true for the instances whose mandatory nodes are visited in the order given. Notice that SPMN_52Order_b has no solution. The time, in Table 4, is measured in seconds. The number of failures means the number of failed alternatives tried before getting the solution.

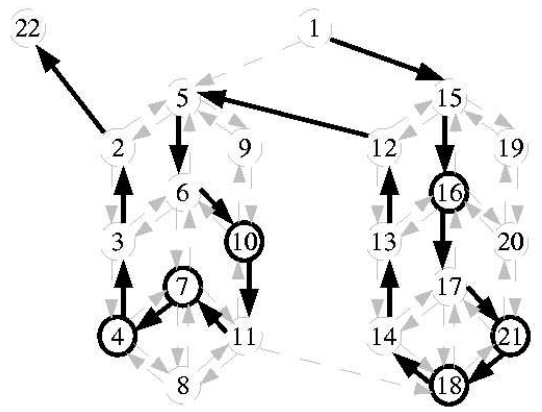

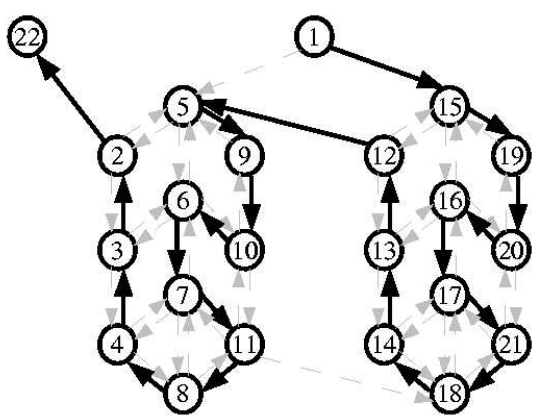

Figure 4: SPMN_22:A path from 1 to 22 visiting 47 Figure 5: SPMN_22full:A path from 1 to 22 visiting 10161821 all the nodes

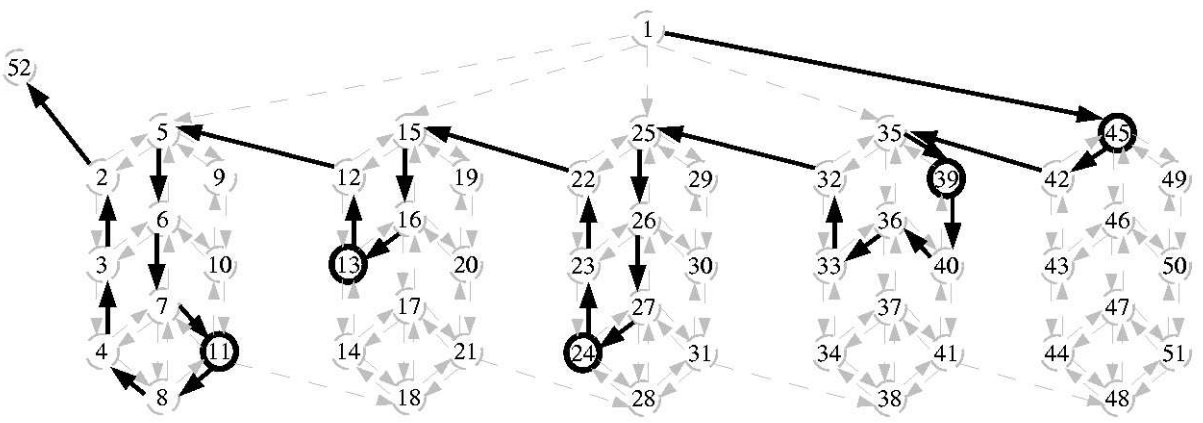

Figure 6: SPMN_52a:A path from 1 to 52 visiting 1113243945

In our experiments, we have made fi ve types of tests: using SPMN without Reachability (column "SPMN"), using SPMN and Reachability but without computing cut nodes nor bridges (column "SPMN+R"), using SPMN and Reachability but without computing bridges (column "SPMN+R+CN"), using SPMN and Reachability but without computing cut nodes (column "SPMN+R+BE"), and using SPMN and Reachability (column "SPMN+R+CN+BE").

As it can be observed in Table 4, we were not able to get a solution for SPMN 22 in less than 30 minutes 


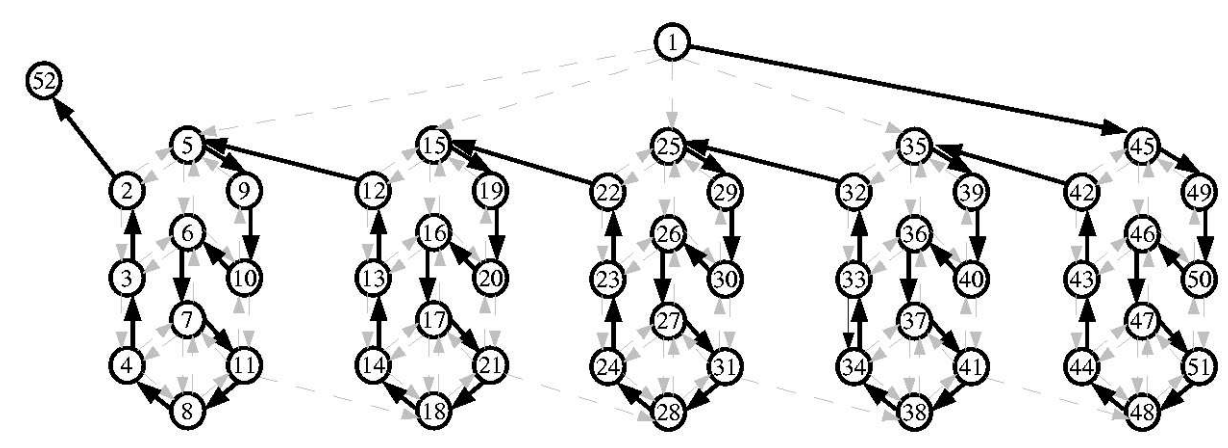

Figure 7: SPMN_52full:A path from 1 to 52 visiting all the nodes

\begin{tabular}{|l|l|l|l|l|l|}
\hline Name & Figure & Source & Destination & Mand. Nodes & Order \\
\hline \hline SPMN_22 & 4 & 1 & 22 & 4710161821 & false \\
\hline SPMN_22full & 5 & 1 & 22 & all & false \\
\hline SPMN_52a & 6 & 1 & 52 & 1113243945 & false \\
\hline SPMN_52b & 6 & 1 & 52 & 45713161922 & false \\
& & & & 2429333639444549 & \\
\hline SPMN_52full & 7 & 1 & 52 & all & false \\
\hline SPMN_52Order_a & 6 & 1 & 52 & 4539241311 & true \\
\hline SPMN_52Order_b & 6 & 1 & 52 & 1113243945 & true \\
\hline
\end{tabular}

\begin{tabular}{|l|l|l|}
\hline Opt. Nodes & Failures & Time \\
\hline 5 & 30 & 89 \\
10 & 42 & 129 \\
15 & 158 & 514 \\
20 & 210 & 693 \\
25 & 330 & 1152 \\
32 & 101 & 399 \\
37 & 100 & 402 \\
42 & 731 & 3518 \\
47 & 598 & 3046 \\
\hline
\end{tabular}

Table 2: Simple Path with mandatory nodes instances

Table 3: Performance with respect to optional nodes

without using Reachability. In fact, we did not even try to solve SPMN_52b without it. However, even though the number of failures is still inferior, the use of Reachability does not save too much time when dealing with mandatory nodes only. This is due to the fact that we are basing our implementation of SPMN on two things: the use AllDiff [17] (that lets us effi ciently remove branches when there is no possibility of associating different successors to the nodes) and the use NoCycle [4] (that avoids re-visiting nodes).

The reason why $S P M N$ does not perform well with optional nodes is because we are no longer able to impose the global AllDiff constrain on the successors of the nodes since we do not know a priori which nodes are going to be used. In fact, one thing that we observed is that the problem tends to be harder to solve when the number of optional nodes increases. In Table 3, all the tests were performed using Reachability on the graph of 52 nodes.

Even though, in SPMN_22, the benefi t caused by the computation of bridges is not that signifi cant, we were not able to obtain a solution for SPMN_52b in less than 30 minutes, while we obtained a solution in 402 seconds by computing bridges. So, even though the computation of bridges is costly, that computation pays off in most of the cases. Nevertheless, cut nodes should be computed in order to profi $t$ from the computation of bridges. 


\begin{tabular}{|l|l|l|l|l|l|l|l|l|l|l|l|l|}
\hline \multicolumn{2}{|c|}{ Problem } & \multicolumn{2}{c|}{ SPMN } & \multicolumn{2}{c|}{ SPMN+R } & \multicolumn{2}{c|}{ SPMN+R+CN } & \multicolumn{2}{c|}{ SPMN+R++BE } & \multicolumn{2}{c|}{ SPMN+R+CN+BE } \\
\hline Instance & Figure & Failures & Time & Failures & Time & Failures & Time & Failures & Time & Failures & Time \\
\hline \hline SPMN_22 & 4 & +130000 & +1800 & 91 & 6.81 & 40 & 6.55 & 70 & 13.76 & 13 & 4.45 \\
\hline SPMN_22full & 5 & 213 & 1.44 & 19 & 0.95 & 0 & 0.42 & 19 & 2.76 & 0 & 1.22 \\
\hline SPMN_52b & - & - & - & +900 & +1800 & +700 & +1800 & +1000 & +1800 & 100 & 402 \\
\hline SPMN_52full & 7 & 3012 & 143 & 774 & 765 & 3 & 8.51 & +700 & 1800 & 3 & 45.03 \\
\hline SPMN_52Order_a & 6 & +12000 & +1800 & 51 & 46.33 & 55 & 81 & 27 & 97 & 16 & 57.07 \\
\hline SPMN_52Order_b & - & +12000 & +1800 & +1500 & +1800 & 81 & 157 & +400 & +1800 & 41 & 117 \\
\hline
\end{tabular}

Table 4: Simple Path with mandatory nodes tests

\subsection{Labeling strategy}

Reachability provides interesting information for implementing smart labeling strategies due to the fact that it associates each node with the set of nodes that it reaches. This information can be used to guide the search in a smart way. For instance, we observed that, when choosing fi rst the node that reaches the most nodes, we obtain paths that minimize the use of optional nodes (as it can be observed in 6).

\subsection{Imposing order on nodes}

An additional feature of Reachability is the suitability for imposing dependencies on nodes (which is a common issue in routing problems). In fact, it might be the case that we have to visit the nodes of the graph in a particular (partial) order.

Our way of forcing a node $i$ to be visited before a node $j$ is by imposing that $i$ reaches $j$ and $j$ does not reach $i$. The tests on the instances SPMN_52Order_a and SPMN_52Order_b show that Reachability takes the most advantage of this information to avoid branches in the search tree with no solution. Notice that we are able to solve SPMN_52Order_a (which is an extension of SPMN_52a) in 57.07 seconds. We are also able to detect the inconsistency of SPMN_52Order_b in 117 seconds.

\section{Related work}

- The cycle constraint of CHIP $[1,2] \operatorname{cycle}\left(N,\left[S_{1}, \ldots, S_{n}\right]\right)$ models the problem of finding $N$ distinct circuits in a directed graph in such a way that each node is visited exactly once. Certainly, Hamiltonian Path can be implemented using this constraint. In fact, [2] shows how this constraint can be used to deal with the Euler knight problem (which is an application of Hamiltonian Path). However, this constraint only covers the case where we are to visit all the nodes of the graph, which is a specific case of Simple Path with mandatory nodes.

- [19] suggests some algorithms for discovering mandatory nodes and non-viable edges in directed acyclic graphs. These algorithms are extended by [3] in order to address directed graphs in general with the notion of strongly connected components and condensed graphs. Nevertheless, examples like SPMN_52a represent tough scenarios for this approach since almost all the nodes are in the same strongly connected component.

- $\mathrm{CP}(\mathrm{Graph})$ introduces a new computation domain focussed on graphs including a new type of variable, graph domain variables, as well as constraints over these variables and their propagators [7, 8]. $\mathrm{CP}($ Graph $)$ also introduces node variables and edge variables, and is integrated with the fi nite 
domain and fi nite set computation domain. Consistency techniques have been developed, graph constraints have been built over the kernel constraints and global constraints have been proposed. $\operatorname{Path}(p, s, d$, maxlength $)$ is one of these global constraints. This constraint is satisfi ed if $p$ is a simple path from $s$ to $d$ of length at most maxlength. Certainly, Simple Path with mandatory nodes can be implemented in terms of Path. However, we still have to compare the performance of Reachability with respect to this approach.

\section{Conclusion and future work}

We presented Reachability: a constrained path propagator that can be used for speeding up constrained path solver. After introducing its semantics and pruning rules, we showed how the use of Reachability can speed up a standard approach for dealing with Simple Path with mandatory nodes.

Our experiments show that the gain is increased with the presence of optional nodes. This is basically because we are no longer able to apply the global AllDiff since we do not know a priori which nodes participate in the path.

From our observations, we infer that the suitability of Reachability is based on the strong pruning that it performs and the information that it provides for implementing smart labeling strategies. We also found that Reachability is appropriate for imposing dependencies on nodes. Certainly, we still have to see whether our conclusions apply to other types of graphs.

It is important to remark that both the computation of cut nodes and the computation of bridges play an essential role in the performance of Reachability. The reason is that each one is able to prune when the other can not. Notice that Figure 2 is a context where the computation of bridges cannot infer anything since there is no bridge. Similarly, Figure 3 represents a context where the computation of bridges discovers more information than the computation of cut nodes.

A drawback of our approach is that each time we compute cut nodes and bridges from scratch, so one of our next tasks is to overcome this limitation. I.e., given a graph $g$, how can we use the fact that the set of cut nodes between $i$ and $j$ is $s$ for recomputing the set of cut nodes between $i$ and $j$ after the removal of some edges? We believe that a dynamic algorithm for computing cut nodes and bridges will improve our performance in a radical way.

As mentioned before, the implementation of Reachability was suggested by a practical problem regarding mission planning in the context of an industrial project. Our future work will concentrate on making propagators like Reachability suitable for non-monotonic environments (i.e., environments where constraints can be removed). Instead of starting from scratch when such changes take place, the pruning previously performed can be used to repair the current pruning.

\section{References}

[1] N. Beldiceanu and E. Contejean. Introducing global constraints in chip, 1994.

[2] E. Bourreau. Traitement de contraintes sur les graphes en programmation par contraintes. Doctoral dissertation, Université Paris, Paris, France, 1999.

[3] H. Cambazard and E. Bourreau. Conception d'une contrainte globale de chemin. In 10e Journées nationales sur la résolution pratique de problèmes NP-complets (JNPC'04), pages 107-121, Angers, France, June 2004.

[4] Y. Caseau and F. Laburthe. Solving small TSPs with constraints. In International Conference on Logic Programming, pages 316-330, 1997. 
[5] T. Cormen, C. Leiserson, and R. Rivest. Introduction to Algorithms. The MIT Press, 1990.

[6] R. Dechter. Constraint Processing. Morgan Kaufmann, 2003.

[7] G. Dooms, Y. Deville, and P. Dupont. Constrained path fi nding in biochemical networks. In 5èmes Journées Ouvertes Biologie Informatique Mathématiques, 2004.

[8] G. Dooms, Y. Deville, and P. Dupont. CP(Graph):introducing a graph computation domain in constraint programming. In CP2005 Proceedings, 2005.

[9] F. Focacci, A. Lodi, and M. Milano. Solving tsp with time windows with constraints. In CLP'99 International Conference on Logic Programming Proceedings, 1999.

[10] M. Garey and D. Johnson. Computers and Intractability: A Guide to the The Theory of NPCompleteness. W. H. Freeman and Company, 1979.

[11] C. Gervet. Interval propagation to reason about sets: Defi nition and implementation of a practical language. CONSTRAINTS journal, 1(3):191-244, 1997.

[12] Mozart Consortium. The Mozart Programming System, version 1.3.0, 2004. Available at http://www.mozart-oz.org/.

[13] T. Müller. Constraint Propagation in Mozart. Doctoral dissertation, Universität des Saarlandes, Naturwissenschaftlich-Technische Fakultät I, Fachrichtung Informatik, Saarbrücken, Germany, 2001.

[14] G. Pesant, M. Gendreau, J. Potvin, and J. Rousseau. An exact constraint logic programming algorithm for the travelling salesman with time windows, 1996.

[15] L. Quesada, P. Van Roy, and Y. Deville. Reachability: a constrained path propagator implemented as a multi-agent system. In CLEI2005 Proceedings, 2005.

[16] L. Quesada, P. Van Roy, and Y. Deville. The reachability propagator. Research Report INFO-2005-07, Université catholique de Louvain, Louvain-la-Neuve, Belgium, 2005.

[17] J. C. Régin. A fi ltering algorithm for constraints of difference in csps. In In Proceedings of the Twelfth National Conference on Artificial Intelligence, pages 362-367, 1994.

[18] C. Schulte. Programming Constraint Services. Doctoral dissertation, Universität des Saarlandes, Naturwissenschaftlich-Technische Fakultät I, Fachrichtung Informatik, Saarbrücken, Germany, 2000.

[19] M. Sellmann. Reduction Techniques in Constraint Programming and Combinatorial Optimization. Doctoral dissertation, University of Paderborn, Paderborn, Germany, 2002.

[20] Y. Shiloach and Y. Perl. Finding two disjoint paths between two pairs of vertices in a graph. Journal of the ACM, 1978.

[21] P. Van Roy and S. Haridi. Concepts, Techniques, and Models of Computer Programming. The MIT Press, 2004. 